\title{
Importância do diagnóstico precoce das cardiopatias congênitas: uma revisão integrativa
}

\author{
Importance of early diagnosis of congenital heart disease: an integrative review
}

Importancia del diagnóstico precoz de las cardiopatías congénitas: una revisión integradora

Isabela Costa Linhares ${ }^{1 *}$, Matheus Hybner Gonçalves ${ }^{1}$, Patrícia Martins Pinto ${ }^{1}$, Manuella da Silva Machado', Mariana Saracino de Almeida', Larissa Sant'Ana Brum¹.

\section{RESUMO}

Objetivo: Construir um conhecimento alicerçado e uniforme para a realização de uma prática médica de qualidade, através de embasamento teórico e análise crítica sobre a importância do diagnóstico precoce das cardiopatias congênitas. Métodos: $O$ presente estudo trata-se de uma revisão literária, integrativa e experimentada por seus autores, cuja elaboração deu-se por meio das seguintes etapas: identificar do tema e selecionar a questão de pesquisa, buscar os artigos nas bases de dados digitais, analisa-los com aplicação de critérios para inclusão e exclusão de estudos, e discussão dos resultados apresentados pelos periódicos. Resultados: Pode-se inferir que a melhor forma de identificação precoce das anomalias cardíacas faz-se por meio do rastreio ultrassonográfico pré-natal e, para um diagnóstico mais preciso e confirmatório, a ecocardiografia fetal. Considerações finais: Confirma-se a relevância do diagnóstico precoce a fim de oportunizar ao paciente um tratamento adequado, qualidade de vida e redução de morbidades, evidenciando a necessidade da inserção de exames como ultrassonografia e ecocardiografia fetal nos programas de triagem pré-natal.

Palavras-chave: Cardiopatias congênitas, Diagnóstico precoce, Triagem pré-natal.

\begin{abstract}
Objective: To build a grounded and uniform knowledge to carry out a medical quality practice, through theoretical foundation and critical analysis on the importance of early diagnosis of congenital heart disease. Methods: This study is a literary review, integrative and experimented by its authors, whose elaboration took place through the following steps: identify the theme and select the research question, search for articles in digital databases, analyze them with the application of criteria for inclusion and exclusion of studies, and discussion of the results obtained by the journals. Results: It can be inferred that the best form of early identification of cardiac anomalies is through prenatal ultrasound screening and, for a more accurate and confirmatory diagnosis, fetal echocardiography. Final considerations: Confirm whether an early diagnosis research is carried out in order to provide the patient with adequate treatment, quality of life and reduction of morbidity, highlighting the need to include tests such as ultrasound and fetal echocardiography in prenatal screening programs.
\end{abstract}

Key words: Congenital heart diseases, Early diagnosis, Prenatal screening.

\section{RESUMEN}

Objetivo: Construir un conocimiento fundamentado y uniforme para realizar una práctica médica de calidad, a través de la base teórica y el análisis crítico sobre la importancia del diagnóstico precoz de las cardiopatías congénitas. Métodos: Este estudio es una revisión literaria, integradora y experimentada por sus autores,

1 Universidade de Vassouras (UV), Vassouras - RJ. *E-mail: isa.costa512@hotmail.com 
cuya elaboración se llevó a cabo a través de los siguientes pasos: identificar el tema y seleccionar la pregunta de investigación, buscar artículos en bases de datos digitales, analizarlos aplicando criterios de inclusión y exclusión. de estudios, y discusión de los resultados obtenidos por las revistas. Resultados: Se puede inferir que la mejor forma de identificación precoz de las anomalías cardíacas es mediante el cribado ecográfico prenatal y, para un diagnóstico más certero y confirmatorio, la ecocardiografía fetal. Consideraciones finales: Confirmar si se realiza una investigación de diagnóstico precoz con el fin de brindar al paciente un tratamiento adecuado, calidad de vida y reducción de la morbilidad, destacando la necesidad de incluir pruebas como la ecografía y la ecocardiografía fetal en los programas de cribado prenatal.

Palabras clave: Cardiopatías congénitas, Diagnóstico precoz, Tamizaje prenatal.

\section{INTRODUÇÃO}

As Cardiopatias Congênitas (CC) são abnormidades decorrentes de falhas anatômicas no coração ou em sua rede, comprometendo sua função. Oferecem grandes riscos à saúde do paciente a partir dos defeitos que podem evoluir de forma gradativa; sejam eles com ou sem sintomas, aumentando as chances de mortalidade. Em alguns casos, interromper a gestação pode ser uma sugestão do próprio obstetra ao identificar sofrimento fetal proveniente de hipóxia ainda no período neonatal (RIVERA IR, et al., 2007; AQUINO TC, et al., 2020).

Cardiopatias congênitas podem ser oriundas de uma associação de fatores genéticos, ambientais, drogas lícitas ou ilícitas, patologias adquiridas durante o período gestacional, no momento em que ocorre a formação do coração; até a oitava semana de gravidez. Malformações cardíacas também são cardiopatias congênitas, porém são anomalias isoladas, prevalentes e responsáveis por 3 a $5 \%$ de mortes de recém-nascidos (BASTOS LF, et al., 2013).

Sabe-se que as doenças cardíacas são divididas em dois grupos que refletem a alteração fisiológica: acianóticos e cianóticos. Os acianóticos são aqueles cujas lesões não cursam com cianose por não causarem obstrução de sangue venoso na circulação sistêmica e são decorrentes de obstrução ao ventrículo direito ou ao ventrículo esquerdo; regurgitação sanguínea ou desvio de sangue da esquerda para a direita (shunt). Por outro lado, as lesões cianóticas são as que cursam com cianose pois o sangue não oxigenado entra na circulação sistêmica, podendo apresentar hipofluxo ou hiperfluxo pulmonar; essas, por sua vez, precisam de intervenção cirúrgica imediata (URAKAWA IT e KOBAYASKI RM, 2012).

Vale considerar que há uma variante quanto à incidência de cardiopatias congênitas entre os países desenvolvidos $(0,8 \%)$ e em desenvolvimento (1,2\%). No Brasil, por exemplo, essa estimativa é de 1 para 100 nascidos vivos, sendo a segunda causa de mortes de acordo com o Sistema de Informação sobre Mortalidade do Ministério da Saúde (SIM), em menores de um ano. Quanto mais severa a cardiopatia, maior é a possibilidade do óbito precoce; por outro lado, as crianças que conseguem sobreviver ao primeiro ano de vida estão sujeitas ao curso de sua enfermidade e aos seus riscos (BASTOS LF, et al., 2013).

O acompanhamento à gestante durante o pré-natal é de suma importância, visto que, ao sinal de uma cardiopatia congênita percebida, preferencialmente durante a gestação e através de exames específicos, favorece o prognóstico e antecipa o planejamento terapêutico mais adequado e assertivo, embora não há ainda clareza quanto aos procedimentos mais empregados para isso. No entanto, aproximadamente $30 \%$ dos recém-nascidos, ao receberem alta, não tem nenhum tipo de diagnóstico e tendem à evolução da doença que venha a ter (SILVA LDC, et al., 2018).

Por apresentar na maioria dos casos tratamento cirúrgico, hoje, a grande preocupação da pediatria com relação as cardiopatias congênitas não é a sua ocorrência em si, mas as consequências advindas dessas anomalias com rápida deterioração clínica e sua alta taxa de mortalidade (URAKAWA IT e KOBAYASKI RM, 2012).

Diante do que foi exposto, este estudo teve como propósito apresentar a importância do diagnóstico precoce para tais patologias a fim de definir o melhor tratamento e maior probabilidade de êxito, no atendimento ao neonato. 


\section{MÉTODOS}

O presente artigo trata-se uma revisão integrativa de literatura especializada, pautada em indícios, com a finalidade de construir um conhecimento fundamentado e uniforme, que venha agregar informações relevantes para uma conduta médica mais eficiente. Os estágios para construção desta revisão foram: definir o tema e apurar a questão de pesquisa, buscando os artigos nas bases de dados digitais, analisar os textos com aplicação de critérios para inclusão e exclusão de estudos e discutir os resultados apresentados pelos periódicos.

A primeira etapa estabeleceu a formulação da hipótese a fim de esclarecer o problema abordado na pesquisa, relacionada ao diagnóstico precoce das cardiopatias congênitas: "qual é a importância do diagnóstico precoce das cardiopatias congênitas?".

A segunda etapa fundamentou-se na busca de artigos publicados no período de 2016 a 2021 cujas bases de dados utilizadas foram Google Acadêmico e Scientific Electronic Library Online (Scielo), em português, e PubMed, em inglês, utilizando os seguintes descritores: importância, diagnóstico precoce, cardiopatias congênitas e triagem neonatal.

No PubMed foram encontrados no total 869 artigos, filtrando os achados para o período de tempo 2016 a 2021, sendo encontrados 111 artigos, sendo selecionados os artigos de acordo com a relevância para o tema. $\mathrm{Na}$ base de dados Scielo as buscas resultaram em 2 artigos; no Google Acadêmico foram encontrados 4510 artigos e, após a aplicação do filtro no período de 2016 a 2021, foi obtido 850 .

$\mathrm{Na}$ terceira etapa, de todos os arquivos encontrados nas três bases de dados pesquisadas, foram excluídos os duplicados e utilizadas apenas 19 publicações, com base nos critérios de inclusão e foram excluídos artigos que tangenciam o tema, bem como os descritores que não estavam correferidos à moção desta pesquisa.

Ademais, todas as informações contidas nos 19 periódicos selecionados foram detalhadamente examinadas com a finalidade de expandir o arcabouço teórico deste artigo. A quarta etapa, por sua vez, constituiu a análise interpretativa de todos os artigos selecionados, lidos e avaliados, bem como a discussão dos resultados apresentados em cada um deles, comprovando a legitimidade e importância do diagnóstico precoce das cardiopatias congênitas (Figura 1).

Figura 1 - Fluxograma da seleção dos estudos para esta revisão.

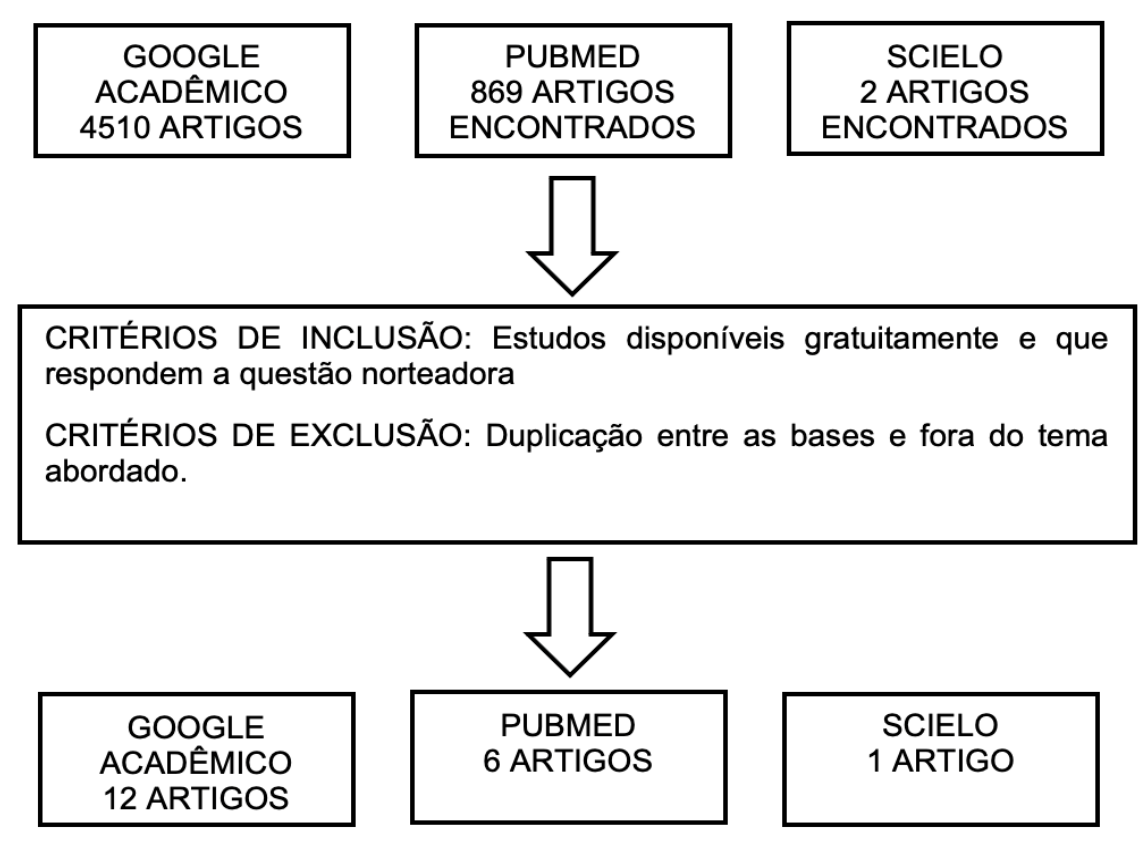

Fonte: Linhares IC, et al., 2021. 


\section{RESULTADOS}

Primeiramente, a partir da seleção de 19 artigos com descritores específicos e identificados como adequados ao que este trabalho se propõe, cada um deles foi arquivado para pesquisa, análise e observação critica. Após leitura minuciosa dos artigos selecionados e acesso aos resumos de todos os exemplares, metodicamente revisados e examinados, apurou-se os pontos mais importantes no que diz respeito à temática abordada.

Tais artigos variam entre os anos de 2006 a 2020, a maioria deste último ano; sendo 11 nacionais, com destaque para região Sul do país, e os demais, internacionais, com ênfase para estudos realizados na China. A grande parte descreve que o diagnóstico precoce das cardiopatias congênitas é o caminho mais eficaz para determinação dos prognósticos, exames específicos e tratamentos mais adequados. Cuidadosamente apurados, pode-se perceber que é de consenso prevalecente que o exame de ecocardiografia fetal tem seu destaque, seguido por outros exames distintivos como ultrassonografia pré-natal, translucência nucal, teste do coraçãozinho (teste de oximetria de pulso), eletrocardiograma, anamnese e exame clínico; e ainda biomarcador cardiovascular e diagnóstico citogenético em outros casos.

Identificar uma malformação pela ultrassonografia depende da capacidade e conhecimento do profissional habilitado para isso. Estudo observacional realizado na Dinamarca infere que as abordagens de triagem durante o pré-natal devem ser garantidas às gestantes bem como exames de rotina podem variar entre os países; sendo que $30,2 \%$ de fetos e crianças com doença cardíaca grave foram diagnosticadas durante 0 período gestacional (LYTZEN R, et al., 2018).

Da mesma maneira, o estudo ecocardiográfico fetal é um procedimento complementar e confirmatório da ultrassonografia, essencial para definir a cardiopatia estrutural e suas alterações funcionais, bem como a necessidade ou não de intervenção terapêutica ainda no período neonatal (CARVALHO SRM, et al., 2006).

Exames ecocardiográficos ainda no período gestacional possuem precisão de 97,7\%, especificidade de $88,9 \%$ e acurácia de $93 \%$ na identificação das cardiopatias, sendo deste total $16,7 \%$ para estudo morfológico e 11,5\% para o obstétrico (PINHEIRO DO, et al., 2019).

Ao diferenciar as cardiopatias congênitas, seja pela ultrassonografia básica ou ampliada, determinou-se que $73 \%$ das anomalias cardíacas pode ser diagnosticada por visão de 4 câmaras e os $30 \%$ restantes pela visão de via de saída, sendo essencial na distinção das cardiopatias. A concordância entre ultrassonografia e ecocardiografia pré-natal e pós-natal é de $87 \%$, de acordo com estudo realizado no Centro de Referência Perinatal Oriente (CERPO) e no Centro Cardiovascular do Hospital Luis Calvo Makenna (HLCM) de Santiago, Chile (MAYORGA C, et al., 2013).

Vale ressaltar que o Teste de Oximetria de Pulso (TOP), apesar de relativamente "novo" no Brasil (sendo determinado pelo Ministério da Saúde como método de Triagem Neonatal apenas em 2014), é uma ferramenta de detecção de anomalias em neonatos supostamente saudáveis. Sua especificidade é de 99,9\% e $75 \%$ de sensibilidade, devendo ser realizado nas primeiras $24 \mathrm{~h}$ de vida (HISHINUMA G, 2017).

Observa-se que as informações obtidas em todos os artigos selecionados complementam-se, evidenciando pouca ou nenhuma discordância, considerando os dados de cada periódico: o método diagnóstico das cardiopatias e perfil epidemiológico analisado, demonstrados através dos dados dispostos no Quadro 1, para título de comparação e exemplificação do que foi dito. 
Quadro 1 - Resultados obtidos após análise dos periódicos.

\begin{tabular}{|c|c|c|c|}
\hline Título & $\begin{array}{l}\text { Autor / Ano de } \\
\text { publicação }\end{array}$ & Diagnóstico das cardiopatias & Perfil epidemiológico \\
\hline $\begin{array}{l}\text { Rastreamento e diagnóstico } \\
\text { ecocardiográfico das arritmias e } \\
\text { cardiopatias congênitas fetais. }\end{array}$ & $\begin{array}{l}\text { CARVALHO } \\
\text { SRM, et al., } \\
\quad 2006 .\end{array}$ & $\begin{array}{l}\text { O diagnóstico foi feito em } 2 \text { níveis. } \\
\text { Um como ultrassonografia } \\
\text { morfológica e outro por } \\
\text { ecocardiograma fetal. }\end{array}$ & $\begin{array}{l}1159 \text { gestantes portadoras de fatores de risco para } \\
\text { alterações cardíacas de seus fetos, selecionados entre } \\
\text { as } 3061 \text { gestantes examinadas no Ambulatório de } \\
\text { Morfologia Fetal (AMOR) do Departamento de } \\
\text { Ginecologia e Obstetrícia da Faculdade de Medicina de } \\
\text { Ribeirão Preto, Universidade de São Paulo, durante o } \\
\text { período de janeiro de } 1997 \text { a dezembro de } 2004 \text {. }\end{array}$ \\
\hline $\begin{array}{l}\text { Cardiopatias congênitas em recém- } \\
\text { nascidos: avaliação da prevalência em um } \\
\text { Hospital de ensino da cidade de Juiz de } \\
\text { Fora. }\end{array}$ & $\begin{array}{l}\text { SANTOS AA, et } \\
\text { al., } 2020 .\end{array}$ & $\begin{array}{l}\text { O diagnóstico foi realizado por meio } \\
\text { de ecocardiograma bidimensional } \\
\text { com Doppler. }\end{array}$ & $\begin{array}{c}77 \text { recém-nascidos com suspeita de CC nascidos no } \\
\text { Hospital Maternidade Therezinha de Jesus (HMTJ) no } \\
\text { período de } 2016 \text { e } 2017 \text {. }\end{array}$ \\
\hline $\begin{array}{c}\text { Perfil epidemiológico das cardiopatias } \\
\text { congênitas em uma maternidade na Serra } \\
\text { Catarinense em } 2016 .\end{array}$ & $\begin{array}{l}\text { ROSA GN, et } \\
\text { al., } 2020 \text {. }\end{array}$ & $\begin{array}{l}\text { O diagnóstico foi determinado por } \\
\text { ecocardiograma transtorácico. }\end{array}$ & $\begin{array}{l}\text { Recém-nascidos com idade de } 0 \text { a } 28 \text { dias, no período } \\
\text { de } 01 \text { de janeiro a } 31 \text { de dezembro de } 2016 \text {. }\end{array}$ \\
\hline $\begin{array}{l}\text { Cardiopatias congênitas em um hospital } \\
\text { pediátrico }\end{array}$ & $\begin{array}{l}\text { FRANCESCHI } \\
\text { J, et al., } 2020 .\end{array}$ & $\begin{array}{l}\text { O diagnóstico foi obtido através de } \\
\text { eletrocardiograma (ECG) e } \\
\text { ecocardiograma (ECO). }\end{array}$ & $\begin{array}{c}\text { Foram selecionados } 163 \text { prontuários os quais os } \\
\text { pacientes realizaram ECO e ECG, sendo } 70 \text { referentes } \\
\text { ao ano de } 2016 \text { e } 93 \text { referentes ao ano de } 2018 \text { no } \\
\text { Hospital Pediátrico da Serra Catarinense. }\end{array}$ \\
\hline $\begin{array}{l}\text { Rastreamento das cardiopatias congênitas } \\
\text { críticas em recém-nascidos assintomáticos } \\
\text { de um hospital de ensino. }\end{array}$ & $\begin{array}{l}\text { HISHINUMA G, } \\
2017 .\end{array}$ & $\begin{array}{l}\text { O diagnóstico foi alcançado através } \\
\text { do teste do coraçãozinho ou teste da } \\
\text { oximetria de pulso e, em caso de } \\
\text { alteração, encaminhamento para } \\
\text { ecocardiografia. }\end{array}$ & $\begin{array}{c}\text { Recém-nascidos internados no alojamento conjunto, } \\
\text { assintomáticos e que realizaram pelo menos um teste } \\
\text { de oximetria de pulso (OP) no período de março de } \\
2013 \text { a dezembro de } 2016 \text { no Hospital Universitário de } \\
\text { Maringá (HUM). }\end{array}$ \\
\hline $\begin{array}{l}\text { Análise comparativa entre ecofuncional por } \\
\text { pediatra e ecocardiografia compreensiva } \\
\text { por cardiologista em unidade de terapia } \\
\text { intensiva pediátrica e neonatal. }\end{array}$ & $\begin{array}{l}\text { TORRES RA, et } \\
\text { al., } 2020 .\end{array}$ & $\begin{array}{l}\text { A ecocardiografia foi indicada para } \\
\text { elucidação diagnóstica. }\end{array}$ & $\begin{array}{c}\text { Um total de } 89 \text { casos foram analisados com admissão } \\
\text { variando de zero dia a } 14 \text { anos internados na UTI } \\
\text { neonatal e pediátrica do Hospital Santa Isabela de Ubá- } \\
\text { MG com indicação clínica para avaliação da existência } \\
\text { de alterações anatômicas. }\end{array}$ \\
\hline
\end{tabular}




\begin{tabular}{|c|c|c|c|}
\hline Título & $\begin{array}{l}\text { Autor / Ano de } \\
\text { publicação }\end{array}$ & Diagnóstico das cardiopatias & Perfil epidemiológico \\
\hline $\begin{array}{l}\text { Prevalência de cardiopatias congênitas por } \\
\text { meio de triagem da oximetria de pulso em } \\
\text { recém- nascidos. }\end{array}$ & $\begin{array}{l}\text { JUNIOR JLS, } \\
\quad 2016 .\end{array}$ & $\begin{array}{l}\text { A oximetria de pulso foi } \\
\text { recomendada como forma de } \\
\text { triagem e a confirmação da doença } \\
\text { com ecocardiograma. }\end{array}$ & $\begin{array}{l}\text { Realizado no período de setembro à novembro de 2015, } \\
\text { com recém-nascidos nas maternidades de } 2 \text { hospitais } \\
\text { privados, sendo } 1 \text { deles conveniado ao SUS (Hospital } \\
\text { dos Usineiros e Hospital Unimed). O tamanho da } \\
\text { amostra foi de } 1047 \text { crianças, com idade gestacional } \\
\text { maior que } 34 \text { semanas e que se apresentavam } \\
\text { saudáveis, recém-nascidos com características } \\
\text { sugestivas de síndrome genética e que tinham mais do } \\
\text { que } 34 \text { semanas de idade gestacional, e gestantes ou } \\
\text { puérperas que concordaram em participar do estudo, }\end{array}$ \\
\hline $\begin{array}{l}\text { Diagnóstico pré-natal e desfechos da } \\
\text { gravidez de } 1.492 \text { fetos com cardiopatia } \\
\text { congênita: papel da consulta } \\
\text { multidisciplinar no diagnóstico pré-natal. }\end{array}$ & $\begin{array}{l}\text { QIU X, et al., } \\
2020 .\end{array}$ & $\begin{array}{l}\text { O rastreamento de CC foi realizado } \\
\text { através da utrassonografia sistêmica } \\
\text { e exame ecocardiográfico fetal } \\
\text { durante } 20-28 \text { semanas de gestação. }\end{array}$ & $\begin{array}{l}67.834 \text { mulheres grávidas que se submeteram a } \\
\text { ultrassonografia fetal e triagem do coração fetal na fase } \\
\text { de } 20-28 \text { semanas entre janeiro de } 2012 \text { e dezembro de } \\
2016 \text { no Fujian Maternity and Child Health Hospital. }\end{array}$ \\
\hline $\begin{array}{l}\text { Avaliação do papel da ultra-sonografia } \\
\text { obstétrica de rotina, no rastreamento pré- } \\
\text { natal das anormalidades cardíacas. }\end{array}$ & $\begin{array}{l}\text { BACALTCHUK } \\
\text { T, } 2001 .\end{array}$ & $\begin{array}{l}\text { A ultrassonografia obstétrica pré- } \\
\text { natal e ecocardigrafia fetal a partir } \\
\text { das } 20 \text { semanas de gestação. }\end{array}$ & $\begin{array}{c}77 \text { neonatos ou lactentes internados no Instituto de } \\
\text { Cardiologia do Rio Grande do Sul/Fundação } \\
\text { Universitária de Cardiologia (IC/FUC) no período de } \\
\text { outubro de } 2000 .\end{array}$ \\
\hline $\begin{array}{l}\text { Papel da ecocardiografia na triagem pré- } \\
\text { natal de doenças cardíacas congênitas e } \\
\text { sua correlação com o resultado pós-natal. }\end{array}$ & $\begin{array}{l}\text { SHARMA S, et } \\
\text { al., } 2017 .\end{array}$ & $\begin{array}{l}\text { Foi realizado ecocardiografia pré- } \\
\text { natal e confirmação do diagnóstico } \\
\text { com ecocardiografia pós-natal. }\end{array}$ & $\begin{array}{c}\text { Este foi um tipo de estudo prospectivo correlativo } \\
\text { realizado ao longo de um período de } 12 \text { meses de } \\
\text { fevereiro de } 2015 \text { a fevereiro de } 2016 \text { em mulheres no } \\
\text { meio do trimestre que frequentaram a clínica pré-natal } \\
\text { no Government Medical College e Rajindra Hospital, } \\
\text { Patiala, Punjab, Índia. } 1200 \text { mulheres grávidas. Período } \\
\text { gestacional de } 20 \text { semanas. }\end{array}$ \\
\hline $\begin{array}{l}\text { Epidemiologia, diagnóstico pré-natal, e } \\
\text { resultados neonatais de defeitos cardíacos } \\
\text { congênitos no leste da China: um estudo } \\
\text { multicêntrico baseado em hospital. }\end{array}$ & $\begin{array}{l}\text { ZHANG X, et } \\
\text { al., } 2020 .\end{array}$ & $\begin{array}{l}\text { O diagnóstico foi obtido por meio da } \\
\text { ecocardiografia fetal entre } 24-28 \\
\text { semanas de gestação. }\end{array}$ & $\begin{array}{c}\text { Mulheres com idade } \leq 20 \text { ou } \geq 35 \text { anos na província de } \\
\text { Zhejiang, China. }\end{array}$ \\
\hline $\begin{array}{c}\text { Análise de Teste Genético e de Desfecho } \\
\text { da Gestação de } 362 \text { Fetos com Cardiopatia } \\
\text { Congênita Identificados por Ultrassom Pré- } \\
\text { Natal }\end{array}$ & $\begin{array}{l}\text { LUO S, et al., } \\
2018 .\end{array}$ & $\begin{array}{l}\text { Ultrassonografia fetal entre } 20-28 \\
\text { semanas e ecocardiograma fetal } \\
\text { posteriormente para confirmação, } \\
\text { incluindo cariotipagem e análise } \\
\text { cromossômica por microarray (CMA). }\end{array}$ & 362 casos de CC entre 2009 e 2016. \\
\hline
\end{tabular}




\begin{tabular}{|c|c|c|c|}
\hline Título & $\begin{array}{l}\text { Autor / Ano de } \\
\text { publicação }\end{array}$ & Diagnóstico das cardiopatias & Perfil epidemiológico \\
\hline $\begin{array}{l}\text { Ecocardiografia fetal precoce: detecção de } \\
\text { cardiopatia congênita e precisão } \\
\text { diagnóstica nas mãos de um programa de } \\
\text { cardiologia fetal experiente. }\end{array}$ & $\begin{array}{l}\text { PIKE Jl, et al., } \\
2014 .\end{array}$ & $\begin{array}{l}\text { O ecocardiograma fetal realizado } \\
\text { ente } 12-16 \text { semanas. }\end{array}$ & $\begin{array}{c}\text { Revisão de ecocardiogramas fetais de } 12-16 \text { semanas } \\
\text { de gestação entre julho de } 2007 \text { e dezembro de } 2010 \text { no } \\
\text { Fetal Heart Programa Children's National Medical } \\
\text { Center. }\end{array}$ \\
\hline $\begin{array}{c}\text { Importância da ultrassonografia } \\
\text { obstétrica de rotina na identificação pré- } \\
\text { natal de defeitos cardíacos congênitos: } \\
\text { eficácia e avaliações de fatores } \\
\text { associados. }\end{array}$ & $\begin{array}{l}\text { POLLI JB, } \\
2016 .\end{array}$ & $\begin{array}{l}\text { O diagnóstico foi realizado através } \\
\text { de ecocardiografia fetal, } \\
\text { translucência nucal e diagnóstico } \\
\text { citogenético pré-natal. }\end{array}$ & $\begin{array}{c}110 \text { pacientes com CC menores de } 2 \text { anos de idade } \\
\text { admitidas pela primeira vez na unidade de terapia } \\
\text { intensiva (UTI) cardíaca do Hospital Criança Santo } \\
\text { Antônio (HCSA), RS. }\end{array}$ \\
\hline $\begin{array}{l}\text { Acurácia do diagnóstico pré-natal de } \\
\text { cardiopatias congênitas. }\end{array}$ & $\begin{array}{l}\text { PINHEIRO DO, } \\
2019 .\end{array}$ & $\begin{array}{l}\text { O diagnóstico foi obtido por meio da } \\
\text { anamnese e ecocardiografia fetal. }\end{array}$ & $\begin{array}{c}96 \text { gestantes atendidas no Serviço de Ecocardiografia } \\
\text { cujos partos ocorreram no Complexo Hospitalar Santa } \\
\text { Casa de Porto Alegre, RS, entre março de } 2013 \text { e } \\
\text { dezembro de } 2015 .\end{array}$ \\
\hline $\begin{array}{l}\text { Cardiopatia congênita: diagnóstico pré- } \\
\text { natal e acompanhamento. }\end{array}$ & $\begin{array}{l}\text { MAYORGA C, } \\
\text { et al., } 2013 .\end{array}$ & $\begin{array}{l}\text { O diagnóstico foi obtido através de } \\
\text { ecocardiograma após realização de } \\
\text { ultrassonografia obstétrica. }\end{array}$ & $\begin{array}{l}568 \text { casos de gestantes cujos fetos foram } \\
\text { diagnosticados com cardiopatias congênitas entre } \\
\text { março de } 2003 \text { e dezembro de } 2011 \text {. }\end{array}$ \\
\hline $\begin{array}{l}\text { O papel da translucência nucal no } \\
\text { rastreamento de cardiopatias congênitas. }\end{array}$ & $\begin{array}{l}\text { BRUNS RF, et } \\
\text { al., } 2006 .\end{array}$ & $\begin{array}{l}\text { Foi utilizada a translucência nucal } \\
\text { para diagnóstico e rastreamento para } \\
\text { cardiopatia congênita. }\end{array}$ & $\begin{array}{c}\text { Gestantes de 11-13 semanas e } 6 \text { dias de idade } \\
\text { gestacional. }\end{array}$ \\
\hline $\begin{array}{l}\text { Avaliação dos níveis de biomarcadores } \\
\text { cardiovasculares circulantes para detecção } \\
\text { precoce de doença cardíaca congênita em } \\
\text { recém-nascidos na Suécia. }\end{array}$ & $\begin{array}{l}\text { CLAUSEN H, et } \\
\text { al., } 2020 .\end{array}$ & $\begin{array}{l}\text { Obteve-se o diagnóstico através de } \\
\text { amostras de sangue seco. }\end{array}$ & $\begin{array}{c}\text { Foram analisadas em } 115 \text { neonatos (81 neonatos } \\
\text { controle e } 34 \text { neonatos cm CC), o teste sozinho } 71 \% \text { de } \\
\text { todos os casos de CC e } 68 \% \text { dos casos críticos após } 2 \\
\text { dias de nascimento. A detecção de qualquer tipo de CC } \\
\text { melhorou para } 82 \% \text { e a detecção de CC crítica } \\
\text { melhorou para } 89 \% \text { quando resultados do teste de } \\
\text { oximetria de pulso e NT-proBNP foram combinados. }\end{array}$ \\
\hline $\begin{array}{l}\text { Principal doença cardíaca congênita } \\
\text { nascida viva na Dinamarca: incidência, } \\
\text { taxa de detecção e taxa de interrupção da } \\
\text { gravidez de } 1996 \text { a } 2013 .\end{array}$ & $\begin{array}{l}\text { LYTZEN R, et } \\
\text { al., } 2018 .\end{array}$ & $\begin{array}{c}\text { Realizaram-se } 2 \text { ultrassonografias: } \\
\text { uma com 11-13 semanas e outra } \\
\text { com } 18-20 \text { semanas. Em caso de } \\
\text { suspeita de CC, ecocardiografia } \\
\text { fetal. }\end{array}$ & $\begin{array}{l}30,2 \% \text { das crianças e fetos com CC importante foram } \\
\text { diagnosticados no pré-natal. }\end{array}$ \\
\hline
\end{tabular}

Fonte: Linhares IC, et al., 2021. 


\section{DISCUSSÃO}

Doenças cardíacas congênitas são anomalias diversas, porém comuns, de maior prevalência e impacto no que diz respeito à mortalidade ou morbidade em neonatos. Responsáveis por um número elevado de malformações desde o período embrionário, podem estar associadas a fatores genéticos ou ambientais, bem como a situações precárias de tratamento e diagnóstico identificadas em alguns países, além de características demográficas, econômicas ou sociais (MAYORGA C, et al., 2013; JUNIOR JLS, 2014).

É imprescindível que um diagnóstico nos primeiros dias de vida seja feito, tendo em vista que as cardiopatias congênitas possuem sinais e sintomas críticos (dependendo do tipo), distintos e, às vezes, de rápida evolução. Vale ressaltar, no entanto, que os recursos tecnológicos e profissionais capacitados são elementos essenciais neste processo (PIKE JI, et al., 2014).

Sabe-se que o número de fetos e/ou crianças com anomalias cardíacas não mudou de forma significativa nos últimos anos. O que se pode observar é que houve um aumento considerável de diagnósticos por conta de avaliações mais rigorosas, precoces e norteadoras; a partir de uma varredura das diversas malformações através de exames disponíveis ou específicos que tendem a evidenciar taxas de detecção e, consequentemente mais estudos para prognósticos positivos (LYTZEN R, et al., 2018).

Decerto, os exames de imagem têm papel fundamental/primordial no acompanhamento gestacional; no entanto, mais importante que o exame em si, o profissional capacitado é de suma importância e necessidade para uma avaliação assertiva no caso de diagnósticos positivos ou tranquilizador se negativo, levando-se em consideração que qualquer suspeita de alteração deve ser seguidamente analisada pelo especialista da área que conduzirá ao tratamento mais adequado, seja ele cirúrgico ou acompanhamento clínico. Dessa forma, um delineamento quanto ao plano de assistência ou tratamento a ser efetuado torna-se heterogêneo, tendo em vista a sua complexidade desde a diagnose, passando pelos cuidados necessários até mesmo ao restabelecimento da saúde do doente (LYTZEN R, et al., 2018).

As manifestações clínicas mais comuns em portadores de cardiopatias congênitas podem ser: cianose, arritmia, taquipneia e sopro cardíaco (não necessariamente nessa ordem). A cianose, é uma característica importante para um diagnóstico diferencial, lembrando que é a primeira evidência para uma suspeita no recém-nascido, independente do quadro clínico, sobretudo se não houver melhora rápida com inalação de oxigênio; cujas causas mais frequentes são: Transposição dos grandes vasos, Tetralogia de Fallot, Atresia da valva pulmonar e Atresia da valva tricúspide (CESARIO MAS, et al., 2020; AMARAL F, et al., 2002).

No caso de arritmia cardíaca, é imprescindível a exclusão de defeitos estruturais associados, podendo ou não ser a sua causa; porém, vale considerar que as principais arritmias que requerem terapêutica neonatal são: Taquicardia paroxística supraventricular (TPSV), Bloqueio atrioventricular congênito completo e Flutter atrial congênito (AMARAL $F$, et al., 2002).

Confirmado por ecocardiograma, na maioria dos casos, a taquipneia/dispneia pode ser considerada como parte de um quadro clínico consoante à gravidade da cardiopatia; suas causas mais frequentes são: Coarctação da aorta, Persistência do canal arterial no prematuro, Estenose valvar Aórtica grave, Síndrome Hipoplásica do coração esquerdo, Tronco arterial comum, Conexão venosa anômala total das veias pulmonares e Atresia tricúspide e Ventrículo único sem estenose pulmonar (AMARAL F, et al., 2002).

Já o sopro cardíaco, permeia uma dúvida: é inocente ou patológico? O sopro inocente é o menos comum no neonato quando comparado a outras faixas etárias enquanto, o sopro patológico não aponta, necessariamente, uma cardiopatia grave, porém a sua ausência também não a exclui. Dentre suas principais etiologias estão: Comunicação Interventricular (CIV), Persistência do canal arterial (PCA), Estenose de valva pulmonar, Estenose de valva aórtica, Sopro inocente, Insuficiência tricúspide funcional e Cardiopatias complexas variadas (AMARAL F, et al., 2002).

Normalmente, estes sintomas tornam-se evidentes ainda no período neonatal e a gravidade dessas ocorrências também acontecem nessa fase. Assim, ao primeiro sinal de uma suspeita clínica, deve-se realizar uma atenção primária de forma acurada, tendo como objetivo identificar de precocemente a cardiopatia em questão. (AMARAL $F$, et al., 2002). 
Crianças portadoras de doenças cardiovasculares devem ser acompanhadas continuamente, desde a assistência clínica usual às intervenções mais complexas, sendo elas atenuantes ou corretivas. Obviamente, em caso de disfunções residuais (insuficiências valvares, comunicações entre cavidades ou arritmias, por exemplo) haverá necessidade de novas abordagens terapêuticas que promovam a reabilitação e promoção da saúde do paciente (SILVA LDC, et al., 2018).

Atualmente, este é um grande desafio para a medicina e expectativa das famílias de crianças portadoras de cardiopatias congênitas. Os protocolos de triagem e programas já implantados pelo Ministério da Saúde, através do Sistema Único de Saúde (SUS), atuam na atenção e identificação das cardiopatias por meio dos exames inseridos nesses protocolos. Porém, não são suficientes para antever o problema, uma vez que exames mais específicos ou antecipatórios são disponibilizados apenas em casos especiais, tais como: malformações fetais, gestantes portadoras de diabetes ou doenças autoimunes, com histórico de cardiopatias, hipertensão arterial ou maior de 35 anos de idade (SILVA LDC, et al., 2018).

Infelizmente, a demanda é maior que a oferta; fatores econômicos e operacionais vão de encontro à necessidade de um diagnóstico precoce a fim de reduzir a evolução de doenças que poderiam primariamente serem identificadas e programadas no que tange ao tratamento mais eficaz (SILVA LDC, et al., 2018).

Periódicos abordados neste estudo, apontam que a ultrassonografia fetal realizada até a $20^{\underline{a}}$ semana da gestação pode evidenciar defeitos na estrutura ou função cardíaca. Por conseguinte, o ecocardiograma fetal é considerado, por seu caráter conclusivo e confirmatório, o padrão ouro nesse diagnóstico. Ainda de forma intrauterina, a Translucência Nucal (TN) é um importante rastreador de doenças cardíacas congênitas devendo ser realizada entre a $11^{a}$ e $13^{\underline{a}}$ semana de gestação em casos de incidência de cardiopatia congênita ou mãe portadora da mesma (SILVA LDC, et al., 2018; JÚNIOR JLS, 2016; BRUNS RF, et al., 2006).

Já após o nascimento, o Teste da Oximetria de Pulso (TOP), também conhecido como Teste do Coraçãozinho, realizado nas primeiras $24 \mathrm{~h} / 48 \mathrm{~h}$ de vida, em todo recém-nascido aparentemente saudável, é ideal para detecção das cardiopatias; seguido por outros exames complementares, em caso positivo, como ecocardiograma, eletrocardiograma, acompanhados pela percepção de sinais clínicos e anamnese. Novos estudos ainda revelam que o uso de macha de sangue seco é utilizado para medir o biomarcador cardiovascular a fim de melhorar a detecção precoce, bem como a análise citogenética cujo objetivo consiste em investigar anomalias cromossômicas com a finalidade de analisar a possível etiologia da cardiopatia. Partindo desse pressuposto, vale ressaltar que o diagnóstico precoce das cardiopatias congênitas é de fato essencial (HISHINUMA G, 2017; JUNIOR JLS, 2016; CLAUSEN H, et al., 2020; POLLI JB, 2016).

Evidencia-se então que, para o sucesso terapêutico, é necessário o cumprimento das diretrizes que otimizam os programas de triagem, comprometimento e participação da equipe multidisciplinar, e integralidade na atenção aos portadores de cardiopatias para validar diagnósticos e terapêuticas, impedindo a ocorrência de eventos inesperados como parada cardíaca, choque e outros, proporcionando qualidade de vida ao paciente (BASTOS LF, et al., 2013; SILVA LDC, et al., 2018).

\section{CONSIDERAÇÕES FINAIS}

Em síntese, é importante salientar que o diagnóstico precoce de doenças cardiovasculares não elimina suas possibilidades, mas pode reduzir significativamente as taxas de morbidade ou mortalidade, garantindo aos pacientes qualidade de vida a partir de tratamentos adequados e assistência necessária. Nesse viés, torna-se relevante considerar que exames como ultrassonografia e ecocardiografia fetal sejam inseridos nos programas de triagem durante a gestação, independente de fatores de risco, possibilitando a condução mais assertiva para cada caso, evitando negligências ou maiores morbidades provenientes de diagnóstico tardio, visando a todo momento a qualidade de vida.

\section{REFERÊNCIAS}

1. AMARAL F, et al. Quando suspeitar de cardiopatia congênita no recém-nascido. Revista Medicina (Ribeirão Preto), 2002; 35(2): 192-197. 
2. AQUINO TC, et al. Aumento da sobrevida de pacientes com cardiopatias congênitas após assistência perinatal e neonatal adequada: relato de caso. Revista Eletrônica Acervo Saúde, 2020; 12(10): e4797.

3. BACALTCHUK T. Avaliação do papel da ultrassonografia obstétrica de rotina, no rastreamento pré-natal das anormalidades cardíacas. Dissertação (Mestrado em Pediatria) - Universidade Federal do Rio Grande do Sul, Porto Alegre, 2001; 101p.

4. BASTOS LF, et al. Perfil clínico e epidemiológico de crianças com cardiopatias congênitas submetidas à cirurgia cardíaca. Journal of Nursing UFPE/Revista de Enfermagem UFPE, 2013; 7(8).

5. BRUNS RF, et al. O papel da translucência nucal no rastreamento de cardiopatias congênitas. Arquivos Brasileiros de Cardiologia, 2006; 87(3): 307-314.

6. CARVALHO SRM, et al. Rastreamento e diagnóstico ecocardiográfico das arritmias e cardiopatias congênitas fetais. Revista Brasileira de Ginecologia e Obstetrícia, 2006; 28(5): 304-309.

7. CESARIO MAS, et al. Mães de crianças com cardiopatia congênita: dúvidas e estratégias de intervenção. Revista Eletrônica Acervo Saúde, 2020;12(5): e2337.

8. CLAUSEN H, et al. Evaluation of circulating cardiovascular biomarker levels for early detection of congenital heart disease in newborns in Sweden. JAMA network open, 2020; 3(12): e2027561-e2027561.

9. ROSA GA, et al. Perfil epidemiológico das cardiopatias congênitas em uma maternidade na serra Catarinense em 2016. Pesquisa, Sociedade e Desenvolvimento, 2020; 9(7): e966975175-e966975175.

10. SANTOS AA, et al. Cardiopatias congênitas em recém-nascidos: avaliação da prevalência em um Hospital de ensino da cidade de Juiz de Fora. Brazilian Journal of Health Review, 2020; 3(6): 15919-15932.

11. FRANCESCHI J, et al. Cardiopatias congênitas em um hospital pediátrico. Research, Society and Development, 2020; 9(6): 84.

12. HISHINUMA G. Rastreamento de cardiopatias congênitas críticas em recém-nascidos assintomáticos de um hospital de ensino. Dissertação (Mestrado em Ciências da Saúde Área de Concentração: Saúde Humana) - Centro de Ciências da Saúde. Universidade Estadual de Maringá, Paraná, 2017; 36 p.

13. LUO S, et al. Análise de Teste Genético e de Desfecho da Gestação de 362 Fetos com Cardiopatia Congênita Identificados por Ultrassom Pré-Natal. Arquivos Brasileiros de Cardiologia, 2018; 111(4): 571-577.

14. LYTZEN R, et al. Principal doença cardíaca congênita nascida viva na Dinamarca: incidência, taxa de detecção e taxa de interrupção da gravidez de 1996 a 2013. JAMA cardiology, 2018; 3(9): 829-837.

15. MAYORGA C, et al. Cardiopatías congénitas: diagnóstico prenatal y seguimiento. Revista chilena de obstetricia y ginecología, 2019; 78(5): 349-356.

16. PINHEIRO DO, et al. Acurácia do diagnóstico pré-natal de cardiopatias congênitas. Revista Brasileira de Ginecologia e Obstetrícia, 2019; 41(1): 11-16.

17. PIKE J, et al. Early fetal echocardiography: congenital heart disease detection and diagnostic accuracy in the hands of an experienced fetal cardiology program. Prenatal diagnosis, 2014; 34(8): 790-796.

18. POLLI JB. Importância da ultrassonografia obstétrica de rotina na identificação pré-natal de defeitos cardíacos congênitos: eficácia e avaliação de fatores associados. Dissertação (Mestrado) - Programa de Pós-Graduação em Patologia. Universidade Federal de Ciências da Saúde de Porto Alegre, Porto Alegre, 2016; 72 p.

19. QIU X, et al. Diagnóstico pré-natal e desfechos da gravidez de 1.492 fetos com cardiopatia congênita: papel da consulta multidisciplinar no diagnóstico pré-natal. Relatórios científicos, 2020; 10(1): 1-11.

20. RIVERA IR, et al. Cardiopatia congênita no recém-nascido: da solicitação do pediatra à avaliação do cardiologista. Arquivos Brasileiros de Cardiologia, 2007; 89(1): 6-10.

21. SHARMA S, et al. Role of echocardiography in prenatal screening of congenital heart diseases and its correlation with postnatal outcome. Journal of clinical and diagnostic research: JCDR, 2017; 11(4): TC12.

22. SILVA LDC, et al. Diagnóstico precoce das cardiopatias congênitas: Uma revisão integrativa. JMPHC| Journal of Management \& Primary Health Care, 2018; 9.

23. JÚNIOR JLS. Prevalência de cardiopatias congênitas por meio de triagem da oximetria de pulso em recém-nascidos. Dissertação (Mestrado) - Pesquisa em Saúde do Centro Universitário CESMAC, Maceió, Alagoas, 2016 ; 63 p.

24. TORRES RA, et al. Análise comparativa entre ecofuncional por pediatra e ecocardiografia compreensiva por cardiologista em unidade de terapia intensiva pediátrica e neonatal. HU Revista, 2020; 46: 1-9.

25. URAKAWA IT, KOBAYASHI RM. Identificação do perfil e diagnósticos de enfermagem do neonato com cardiopatia congênita. Revista de Pesquisa Cuidado é Fundamental Online, 2012; 4(4): 3118-3124.

26. ZHANG $X$, et al. Epidemiologia, diagnóstico pré-natal e resultados neonatais de defeitos cardíacos congênitos no leste da China: um estudo multicêntrico baseado em hospital. BMC pediatrics, 2020; 20(1): 1-8. 